\title{
Blade Tip-Vortices of a Four-Bladed Rotor with Axial Inflow
}

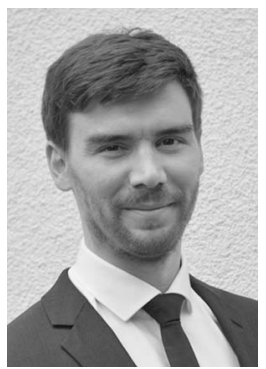

Andreas Goerttler* Research Scientist

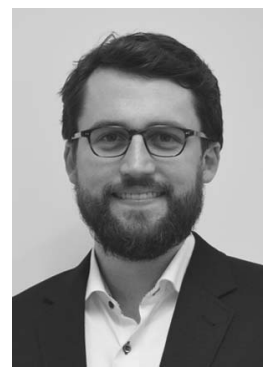

Johannes N. Braukmann Research Scientist

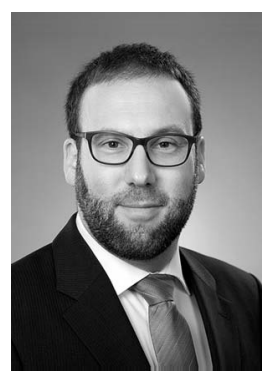

C. Christian Wolf Research Scientist

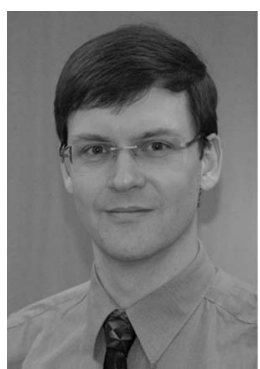

Anthony D. Gardner Research Scientist

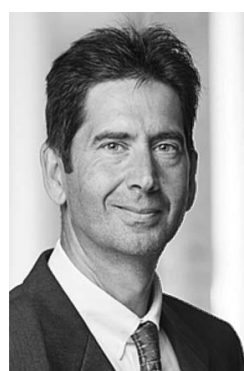

Markus Raffel Research Scientist

German Aerospace Center (DLR), Göttingen, Germany

The vortex system of four rotating and pitching DSA-9A blades was examined numerically and experimentally. Numerical computations were performed using German Aerospace Center (DLR)'s finite-volume solver TAU and were validated against experimental data gathered using particle image velocimetry carried out at the rotor test facility (RTG) in Göttingen. Algorithms deriving the vortex position, swirl velocity, circulation, and core radius were implemented. Hover-like conditions with a fixed blade pitch were analyzed giving further physical insights of the static vortex system. These results are used to understand the vortex development for the unsteady pitching conditions, which can be described as a superpositioning of static vortex states. The use of a zonal detached-eddy simulations approach improved physical modeling of the vortex development by resolving finer scales than URANS. Trimmed cases agree well with differences less than $0.5 \%$ in the circulation and swirl velocity.

\section{Nomenclature}

$a, b$
$C_{T}$
$c$
$M$
$N_{\text {inner }}$
$N_{\text {iter }}$
$R$
$R e$
$R_{\rho}$
$r$
$r_{c}$
$r_{v}$
$\bar{r}_{c}$
$\bar{r}_{v}$
$t / T$
$U$
$u_{p}$
$\bar{u}_{p}$
$v_{\phi}$
$\bar{v}_{\phi}$
$x_{p}, y_{p}, z_{p}$
$y^{+}$
$\Gamma$
$\bar{\Gamma}$

Mach number, $M_{\text {tip }}=0.28$

inner iterations

density-residual

radial distance, $\mathrm{m}$

vortex core radius, $\mathrm{m}$

normalized period time

velocity, $\mathrm{m} / \mathrm{s}$

downward direction, $\mathrm{m}$

circulation, $\mathrm{m}^{2} / \mathrm{s}$ major and minor axis of an ellipse

blade thrust coefficient, $C_{T}=F_{z} /\left(\rho_{\infty}(\Omega R)^{2} \pi R^{2}\right)$

blade chord length, $c=0.072 \mathrm{~m}$

total number of inner iterations

rotor blade radius, $R=0.65 \mathrm{~m}$

Reynolds number based on chord, $R e_{\text {tip }}=470,000$

vortex coordinate in radial direction, $\mathrm{m}$

normalized vortex core size, $\bar{r}_{c}=r_{c} / c$

normalized radial distance, $\bar{r}_{v}=r_{v} / c$

axial velocity along $x_{p}, \mathrm{~m} / \mathrm{s}$

normalized axial velocity, $\bar{u}_{\mathrm{p}}=u_{\mathrm{p}} / U_{\text {tip }}$

swirl velocity along $\phi_{v}, \mathrm{~m} / \mathrm{s}$

normalized swirl velocity, $\bar{v}_{\phi}=v_{\phi} / U_{\text {tip }}$

vortex cut plane coordinates in axial, outboard, and

dimensionless wall distance

normalized circulation, $\bar{\Gamma}=\Gamma /\left(c \cdot U_{\text {tip }}\right)$

*Corresponding author; email: andreas.goerttler@dlr.de.

Presented at the Vertical Flight Society 75th Annual Forum \& Technology Display, Philadelphia, PA, May 13-16, 2019. Manuscript received November 2019; accepted June 2020.

$\lambda_{2}$

$\Delta b$

$\Delta s$

$\Theta_{r}$

$\sigma$

$\phi_{v}$

$\Psi$

$\Psi_{V}$

$\Omega$

$\uparrow$

$\downarrow$

Every lift generating wing and rotating blade develops vortices, which are transported away with the freestream velocity for fixed wing aircraft. The vortex generation and propagation on helicopters is of particular interest, since the rapidly varying lift with time results in a complex vortex form. The vortices interact with other components of the aircraft, with potentially significant consequences for the aerodynamic performance, aeroacoustic performance, and vibration. The vortex rollup, as the vortex moves downstream, is a challenging numerical problem, due to a tendency to unphysical dissipation if the computation is underresolved or particular numerical models are used. Thus, the vortex rollup and changes in swirling strength and core radius are important calibration parameters for computational fluid dynamics (CFD) codes attempting to reproduce vortex interactions.

Early experimental work with pressure probes by Chow et al. (Ref. 2) and Birch et al. (Ref. 3) and hot-wire probes by Devenport et al. (Ref. 4) indicated an almost complete rollup after one chord length downstream on fixed wings. In particular, secondary and tertiary vortices dependent on certain blade tip geometries merge into the primary vortex within short distance. This data of the vortex rollup are still widely used by 
other researchers (Refs. 5,6); however, the measurement of vortices with a probe is subject to a number of systematic errors, particularly regarding the probe positioning. Those techniques are intrusive, thus changing the flow field. Furthermore, they are only able to capture one point at a time. Especially, averaging highly dynamic results introduces errors. An alternative is to use particle seeding, as for instance Gray (Ref. 7), who visualized the vortex of a helicopter rotor with smoke, Leishman et al. (Ref. 8), who used laser Doppler velocimetry (LDV), or Heineck et al. (Ref. 9), who used particle image velocimetry (PIV), to evaluate the evolution of the tip vortex of a hovering rotor. Detached-eddy simulations (DES) on a pitching, fixed airfoil by Mohamed et al. (Ref. 10) showed that the DES model outperformed Reynolds-averaged NavierStokes (RANS) models in matching the circulation and the radius of the blade-tip vortex. Garmann and Visbal (Refs. 11-13) performed extensive numerical simulations of the NACA0012 profile. They compared the vortex at fixed and various oscillating conditions at up to three chord lengths downstream observing an orbital rotation of the vortex core. The numerical and experimental study of Duraisamy et al. (Ref. 14) examined the formation of the blade-tip vortex of a rotating wing at $R e_{\text {tip }}=272,000$ identifying the production and merging of multiple vortex structures. Lietzau et al. (Ref. 15) compared numerical and experimental results of a single-bladed hovering rotor. They noted that the core radii of vortices at a young wake age $\left(\Psi_{V}<15^{\circ}\right)$ were consistently overpredicted by all investigated grids, grid stencils, and turbulent models. The peak swirlvelocity showed a better agreement with the PIV measurements leading to good agreement of the circulation of the vortex. There are several investigations trying to find methods to reduce or diffuse the blade-tip vortex (see Ref. 16).

Jain (Ref. 17) undertook hover simulations, investigating the effect of grid and solver settings on the vortex development. It was noted that the core radius and circulation were sensitive to the approach used but the performance, blade loads, and tip-vortex wake positions showed little variation. The best resolved tip vortex is derived by the finest grid and DES modeling in the wake. In contrast, Richez (Ref. 18) used a secondorder RANS approach to simulate the blade-vortex interaction leading to dynamic stall, with considerable success in reproducing wind tunnel data of the test case. Chaderjian (Ref. 19) used a delayed detached eddy simulation (DDES) approach to investigate a UH60-A in forward-flight, modeling the rotor-wake interactions accurately. However, Jayaraman and Potsdam (Ref. 20), who used a similar approach to investigate the effects of the fuselage and wind tunnel on the wake, noted that even when the vortex properties are well predicted, that a misprediction of the wake trajectory can have a critical effect on the prediction of the interaction aerodynamics.

Wolf et al. (Ref. 21) recently analyzed the vortex of a finite wing, with the DSA-9A airfoil. They noted that the tip vortex continued becoming stronger in the upstroke of the pitching cycle even after dynamic stall. Kaufmann et al. (Ref. 22), reproduced the experimental circulation for the very young vortex $(\Delta x / c<0.25)$ with a sufficiently fine grid $(s / c<$ 0.0075) using RANS.

The focus of this study is a thorough numerical investigation of the evolution and convection of the blade-tip vortex. Contrary to many other studies, rotating blades with dynamic pitch inputs are considered. A comparison with experimental results of collective cases validates the numerical approach and allows for a novel analysis of a rotating dynamic pitch case based on numerical simulations only. Some experimental results are already published by Wolf et al. (Ref. 23) and Braukmann et al. (Ref. 24). Prior numerical analysis has been done by Goerttler et al. (Ref. 25) with a two-bladed rotor. The use of a zonal DES approach ensures improved vortex conservation. This numerical study treats five collective cases $\Theta_{r}=\left[18^{\circ} ; 21^{\circ} ; 24^{\circ} ; 27^{\circ} ; 30^{\circ}\right]$ and a dynamic pitch case as shown in Table 1 . The mean angle and the amplitude $\left(\Theta_{r}=24^{\circ} \pm 6^{\circ}\right)$ are chosen
Table 1. Root angle $\Theta_{r}$ of investigated and shown cases

\begin{tabular}{lcc}
\hline \hline & CFD & Experiments \\
\hline Static (no pitching) & $18^{\circ} ; 21^{\circ}$ & $18^{\circ} ; 21^{\circ} ; 22.5^{\circ}$ \\
Unsteady (pitching) & $24^{\circ} ; 27^{\circ} ; 30^{\circ}$ & $24^{\circ} ; 27^{\circ} ; 30^{\circ}$ \\
\hline \hline
\end{tabular}

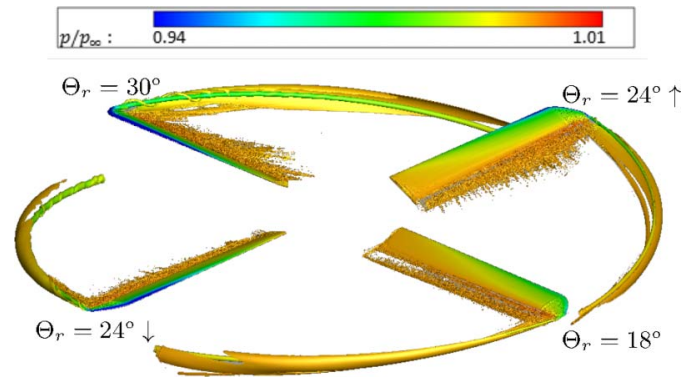

Fig. 1. Isosurface of $\lambda_{2}$ highlights the vortex system of an unsteady pitching motion $\Theta_{r}=24^{\circ} \pm 6^{\circ}, \lambda_{2}=-1000$.

to cover the minimum and maximum of the collective cases, allowing a new, detailed comparison.

\section{Computational Setup}

The DLR-TAU code was used for the numerical simulations. The node-based unstructured finite-volume solver can treat grids with a range of elements including prisms, pyramids, tetrahedra, and hexahedra. The grid is constructed using CENTAUR ${ }^{\mathrm{TM}}$ to have rotor blade grids which utilized a structured prismatic boundary layer region with 30 prism in the direction normal to the surface, a stretching factor of 1.26 , and the first cell height is set to $y^{+} \sim \mathcal{O}(1)$. The surface grid is restricted to a maximum of $\Delta s / c=0.02$, reducing to $\Delta s / c=0.004$ at the leading and trailing edges. Outside of the boundary layer region, a tetrahedral grid is used to a distance of $x / c=1.25$, where the blade grids end. A refinement of the tetrahedral grid to $\Delta s / c=0.004$ in the blade tip region captures the blade-tip vortex and correctly propagates it from the surface into the volume grid, as shown in Ref. 25 . Once in the volume flow, a fine tetrahedral grid $(\Delta s / c=0.005)$ transports the vortex to the edge of the blade grid. Each of the four blade grids has 9.5 million points. The blades are moved as rigid bodies with either prescribed static pitch or sinusoidal cyclic pitch depending on the test case.

Figure 1 shows a visualization for the pitching cycle $\left(\Theta_{r}=24^{\circ} \pm 6^{\circ}\right)$ reaching the highest angle of incidence at the root of $\Theta_{r}=30^{\circ}$ in the upper left corner. For simplicity, only vortices up to the wake age of $\Psi_{V}=90^{\circ}$ are shown in this figure. A variation in both the blade flow and the vortex structures produced can be clearly seen by the isosurface of the $\lambda_{2}$ criterion. In this paper, this cyclic pitching case and collective cases are considered, as shown in Table 1.

The four identical blade grids of the four-bladed RTG rotor are embedded in a background grid which primarily uses tetrahedral cells and has a total size of 48 million points. The background grid has a far-field freestream external boundary implemented as a spherical computational domain with a radius of 900 blade spans. The data exchange between the blade grids and the background grid is by the overset grid (Chimera) method using a three-cell interpolation region, which also transports the second-order flow from the blade grids to the edges of the explicit cutouts in the background grid. With this technique, it is possible to control and rotate each grid independently, as a reduction due to periodicity is not 


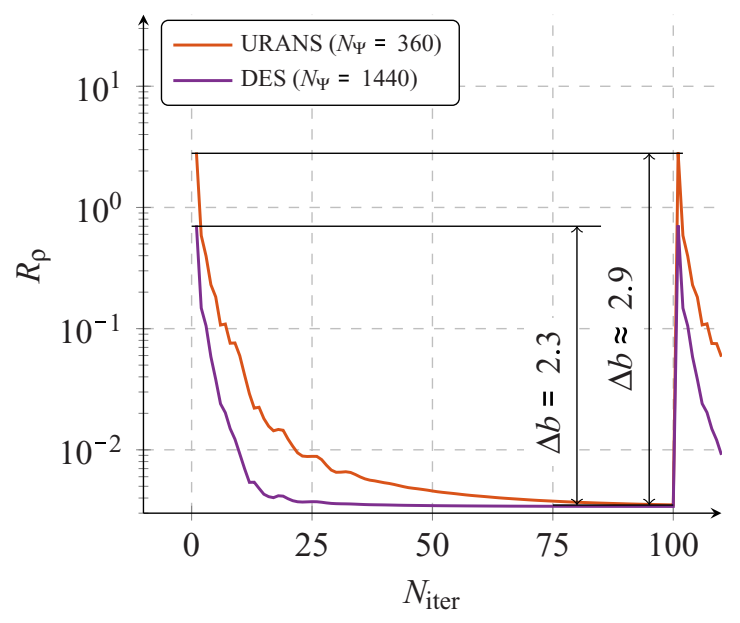

Fig. 2. Density residual of different parameter settings.

feasible with this cyclic pitching case. The tetrahedral background grid had edge lengths of $s / c=0.015$ near the blades, expanding to $s / c=0.2$ one rotor radius downstream of the rotor. A curved, structured grid was introduced into the background grid following the expected path of the blade-tip vortex. This grid was a block-structured H-grid, constructed so that the grid axes were normal and tangential to the vortex core trajectory at each point. The grid had a spacing of $s / c=0.0034$ normal to the vortex core propagation direction near the blade, increasing to $s / c=0.0091$ at a vortex age of $\Psi_{v}=90^{\circ}$. The grid in the vortex core propagation direction was 6:1 coarser than in the normal direction. The background grid with the explicit cutout holes is rotated with the blades, so that in the frame of the background grid the blades have only a pitching motion.

The computations used 1440 timesteps per period, with at least 50 inner iterations per timestep. All shown static simulations have been computed with 60 coarse periods with 36 timesteps per period, followed by two fine periods. The cyclic simulations have been computed with 60 coarse periods, 10 intermediate periods with 360 timesteps per period, and three fine periods with 100 inner iterations per timestep. The periodicity of the final solution was used as the time convergence criterion. The Menter shear-stress transport (SST) model (Ref. 26) is utilized to close the URANS equations. In the region of the blade-tip vortex and away from the boundary layer, a large eddy simulation (LES) model is used (Ref. 27), and in all other regions a conventional SST turbulence model is used. The LES model uses a vorticity-sensitive volume filter to reduce the gray area at the hybrid RANS/LES interface on anisotropic grids by accounting only for the grid spacing in the plane normal to the local vorticity vector. In that plane, the filter reduces to a volume-like formulation. The Smagorinsky subgrid scale model (Ref. 28) is used, with the default Smagorinsky coefficient of 0.17. Compared to RANS, the LES formulation resolves more scales instead of modeling. Especially, the scales which are responsible for viscous dissipation are modeled within the RANS approach leading to less accurate or more numerical dissipated solutions than LES. The computations are second order in space and time; the inviscid fluxes are solved by a central method.

Convergence. All simulations were done with a dual-time stepping approach, which uses physical time steps and inner iterations. Figure 2 shows the density residual for two different numbers of physical time steps. $N_{\Psi}=360$ time steps lead to a slightly higher reduction of 2.9 orders of magnitude since the density residual at the start of each time step is higher. A higher number of time steps, $N_{\Psi}=1440$, results in a

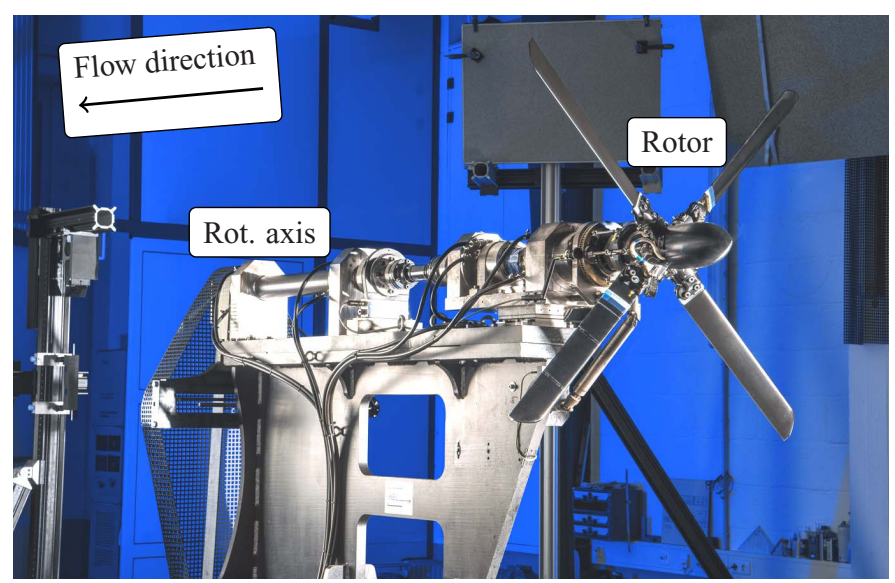

Fig. 3. Overview of the RTG.

reduction of 2.3 orders of magnitude. This study concludes that a sufficiently time converged solution is reached after approximately 30 inner iterations. Chaderjian and Buning (Ref. 29) consider a density residual reduction by $\Delta b=2.2$ as time converged. The above results therefore strongly suggest being time converged.

\section{Experimental Setup}

Figure 3 shows the horizontally mounted rotor at the rotor test facility (RTG) in Göttingen (Ref. 30). An Eiffel-type wind tunnel produces a slow uniform flow of $U_{\infty}=2.24 \mathrm{~m} / \mathrm{s}$. The rotor is mounted horizontally, with the wind tunnel inflow axially from the side. The wakes are propagated out of the room through open doors, avoiding recirculation. The fourbladed rotor has a rotational frequency of $23.6 \mathrm{~Hz}$, a chord length of $c=0.072 \mathrm{~m}$, a blade radius of $R=0.65 \mathrm{~m}$, resulting in a tip Reynolds number of $R e_{\text {tip }}=470,000$ and tip Mach number of $M_{\text {tip }}=0.28$. Each of the four blades has a DSA-9A airfoil (a 9\% helicopter airfoil) with a negative linear twist of $-9.3^{\circ}$ along its span. The rotor blades have no sweep or anhedral, but do have a parabolic (SPP8) tip shape. The rotor can be operated with high cyclic pitch, allowing the creation of unsteady aerodynamic phenomena which are relevant to helicopters.

First experimental results at the rotor test facility (RTG) at the DLR in Göttingen were presented by Schwermer et al. (Ref. 30) who analyzed dynamic stall experimentally. They studied the flow field with PIV of a complete pitching cycle and detected the position of the strongest dynamic stall to be around $80 \%$ radius. The corresponding numerical investigations of the stiff blades have been carried out by Letzgus et al. (Ref. 31), resulting in good agreement with the experiment. The PIV setup used in the current study is similar to that described by Wolf et al. (Ref. 23) and Braukmann et al. (Ref. 24). Stereo-PIV provided the three-component velocity vectors in a measurement plane which is approximately normal to the axes of the blade-tip vortices, enabling a study of both swirl velocity and circulation. The region of interest extends $1.8 c$ in the downstream direction and $2.1 c$ in the inboard direction as shown in Fig. 4. The PIV data were evaluated using iterative crosscorrelation algorithms and the commercial software LaVision DaVis 8.4. The resolution of the final grid is about 4.2 data points per millimeter.

\section{Vortex Analysis}

The vortex characteristics can be investigated from the completed CFD volume solution, analogous to the PIV analysis presented by other authors (Refs. 21, 24, 25). Two-dimensional (2D) cuts are extracted 


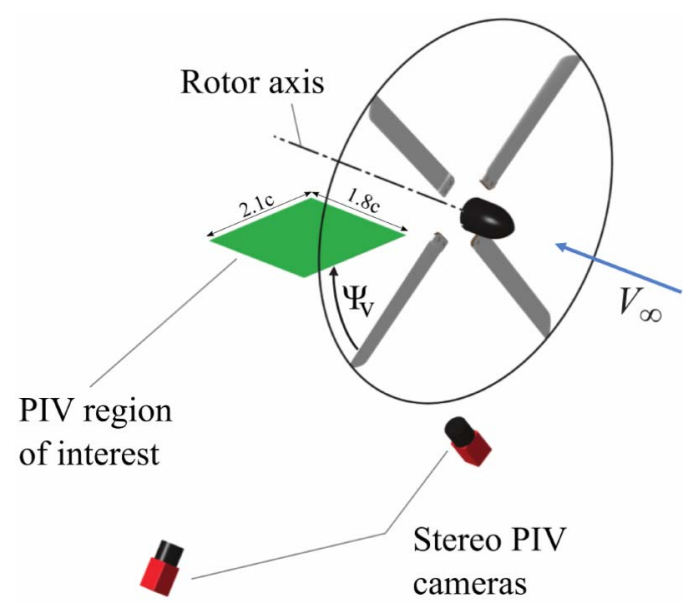

Fig. 4. Sketch of the stereoscopic PIV setup (adapted from Ref. 23).

normal to the path of the blade-tip vortex, similar to PIV planes. The center of the vortex core is defined by the centroid of the lowest $\lambda_{2}$ value. This criterion derives the eigenvalues of a matrix consisting of the squared symmetric and antisymmetric matrix of the gradient velocity tensor (see Ref. 1). This method is chosen, since it captures the pressure minimum. Ordered by value the second eigenvalue indicates a region of a vortex, since two negative eigenvalues are a sufficient characteristic. For the analysis, only negative values are taken into account. Radial cuts are then extracted, radiating star-like from the center with a resolution of $\Delta \Psi=2^{\circ}$ (180 cuts). This is similar to an interpolation of the domain onto a polar coordinate system defined by the vortex core. The vortex core radius is determined by extracting the position of the peak swirl-velocity, and the information of all cuts defines the vortex-core shape.

The bound circulation distribution on the blade is determined by the integration of the surface pressure distribution at each radial station and azimuthal position. This integration yields the lift per unit span. Then, the circulation is estimated with the Kutta-Joukowski theorem (see Ref. 32), which relates the lift of a 2D airfoil to the circulation. Strictly speaking, this theorem is only correct for steady and inviscid flows, but it still gives a useful approximation of the bound circulation on the blade for the relatively low reduced frequencies considered. The radial distribution of the circulation can be integrated to give an estimate of the strength of the departing blade-tip vortex and for wings the vortex moves to the location of the spanwise centroid of vorticity (Refs. 32,33).

The analysis of the unsteady vortex is rather difficult since at each time instant the vortex line consists of a combination of vortex slices of different ages, which were produced at different blade flow conditions. Similarly, following a single vortex slice at a constant distance behind the blade in time will result in a varying vortex which is related to the blade flow a small time beforehand. The propagation rate was simplified assuming that the vortex slice stayed at a constant azimuthal angle in the laboratory frame, with propagation downward and toward the rotor axis.

Figure 5 shows three comparison methods to analyze the influence of the dynamic pitching motion $\left(\Theta_{r}=24^{\circ} \pm 6^{\circ}\right)$ on the wake. On the horizontal axis, there is the azimuthal position of the wake which corresponds to a certain angle of incidence during vortex creation (see upper axis). When pointing along the $\Psi=180^{\circ}$ axis, each blade is always inclined with the maximum angle of $\Theta_{r, \max }=30^{\circ}$. The vertical axis displays the increasing wake age of $0^{\circ}-90^{\circ}$ from top to bottom. Every slice of the wake has a wake age, which is defined by the distance to the blade which created the vortex. Depending on the normalized time and the corresponding position of the rotor, the wake has been created at different blade angles. The horizontal axes, therefore, display

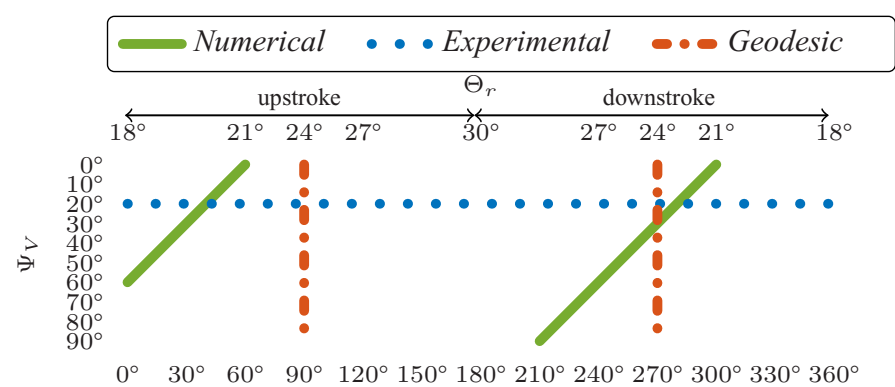

$\Psi$

Fig. 5. Table of visualization approaches depending on wake age and azimuthal position.

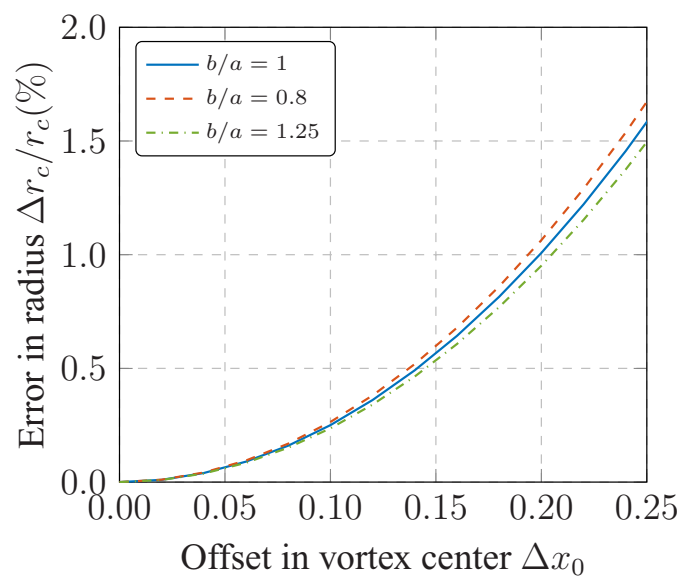

Fig. 6. Influence of offset in center position on radius.

the situation during creation of the vortex. The two parameters wake age and azimuthal position suffice to describe this 2D representation. The three colored lines each represent a different way of looking at the vortex system. The numerical approach supplies solutions of a fixed time, which are presented by the green lines (C). If the vortex at a constant wake age is considered, then many instantaneous solutions are required and the blue line $(\bullet \bullet)\left(\Psi=20^{\circ}\right)$ results. The final approach follows the evolution of a vortex slice produced at a particular instant as it convects downstream of the blade, producing the vertical red line (॰०) in Fig. 5. The different visualization techniques do not show different data but connect different wakes together. Some insights are only apparent with one or the other technique. When cases with collective pitch only are considered, the numerical and geodesic approach fall onto each other. Only the wake age remains as describing parameter.

\section{Error Analysis}

The vortices are detected by extracting $2 \mathrm{D}$ cuts normal to the path of the blade-tip vortex and then seeking the centroid of the area weighted by the $\lambda_{2}$ value. The data transformation into the vortex coordinate system is potentially a source of error. However, Fig. 6 shows that even if the center has an offset of $25 \%$ toward one edge the error of the mean core radius is below $1 \%$. This computation depends on the aspect ratio of the long and short axes of the elliptical vortex $b / a$. For a given offset of $10 \%$ in the center position, the error of the radius stays below $0.25 \%$ and an error of $1 \%$ is reached for an offset of $20 \%$. There are many other techniques to detect the center discussed in the literature with negligible differences (see Ref. 34). 


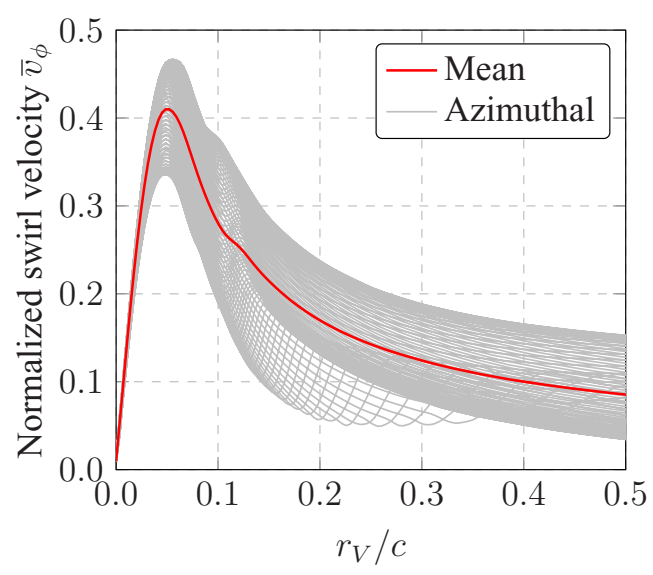

Fig. 7. Azimuthal variation of swirl velocity, $\left(\Theta_{r}=24^{\circ} ; \Psi_{V}=10^{\circ}\right)$.

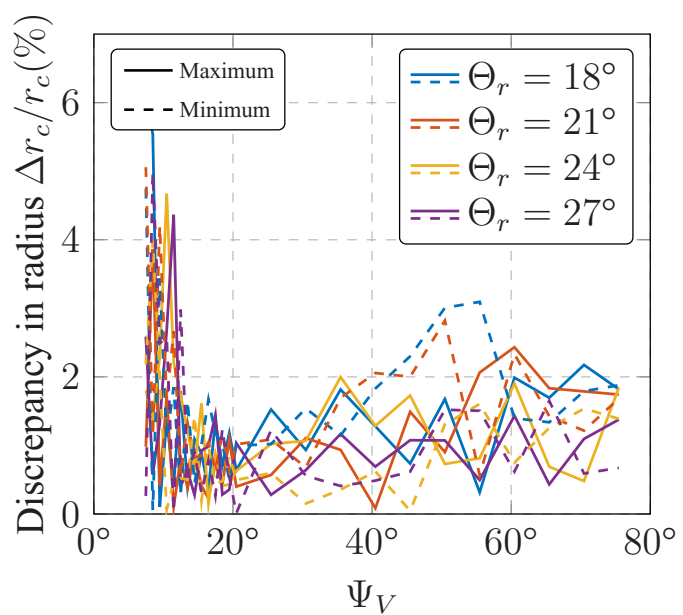

Fig. 8. Discrepancy due to two orthogonal cuts (-- = maximum; - - = = minimum).

Figure 7 shows the azimuthal variation of the swirl velocity of a blade-tip vortex at $\Psi_{v}=10^{\circ}$ with an angle of incidence of $\Theta_{r}=24^{\circ}$. The computation of the swirl velocity requires a correction of the convection velocity of the vortex. The correction has no influence on the mean swirl velocity; however, the peak of the averaged swirl velocity is affected. Additionally, a particular problem is the interaction between the vortex and the wake sheet of the blade, seen as the downward bump in Fig. 7. We use 90 through cut positions (180 star-cuts), but Ramasamy et al. (Ref. 35) suggest that extracting two through-cuts (four star-cuts) and then averaging the four values of the radius core provides a good estimate of the mean radius. Figure 8 shows the error due to only taking two cuts for $\Theta_{r}=24^{\circ}-6^{\circ} \cos (2 \pi t / T)$, with some cut directions overpredicting the radius and some underpredicting. It can be seen that the maximum over- or underprediction in radius is less than $3 \%$ for wake ages older than $\Psi_{v}=20^{\circ}$. For very young wake ages the error can exceed $5 \%$.

The experimental uncertainty is well documented by Wolf et al. in Ref. 23.

\section{Results}

This section presents the outcome of the simulations with five constant pitch angles $\Theta_{r}=\left[18^{\circ}, 21^{\circ}, 24^{\circ}, 27^{\circ}, 30^{\circ}\right]$, with focus on the vortex generation and propagation. Later, the results of the correspond-

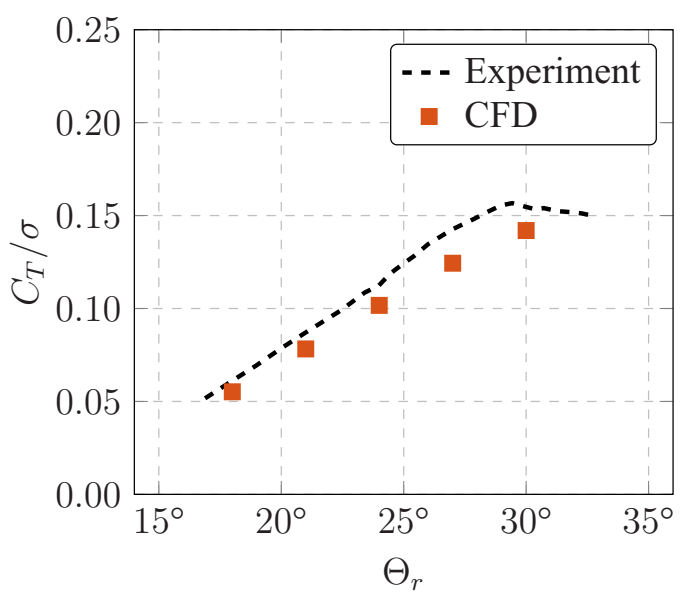

Fig. 9. Comparison of thrust coefficient.

ing pitching simulation with $\Theta_{r}=24^{\circ}-6^{\circ} \cos (2 \pi t / T)$ are compared. Figure 9 displays that the thrust of the rotor is underpredicted. Additionally, it shows that the static stall angle of the rotor in the experiments is $\Theta_{r}=29^{\circ}$, and so the computation at $\Theta_{r}=30^{\circ}$ can be expected to show stalled flow.

Figure 10 displays the $\lambda_{2}$ isosurface of five different light climb (hover with axial inflow) simulations with different collective pitch angles. The isosurfaces are colored with the normalized pressure, indicating the suction peak on the blade and the decrease of pressure with increasing angle of incidence. The creation process of the vortex starts at the leading edge of the tip, forming a slender vortex. The blade-tip vortex grows with an increasing angle of incidence, staying slender except at the highest angle $\left(\Theta_{r}=30^{\circ}\right)$, which shows a radically expanded vortex covering $\Delta r / R \approx 5 \%$. This result has been shown to be associated with separated flow (see Ref. 36), which is in agreement with the expectation from the thrust polar.

\section{Comparison between DES and URANS methods}

Quantitative results of the tip vortex at a young wake age of $\Psi=10^{\circ}$ and $\Psi=25^{\circ}$ are shown in Fig. 11 for collective pitch angles of $\Theta_{r}=$ $22.5^{\circ}$ and $\Theta_{r}=24^{\circ}$. The radial distributions of the swirl velocity clearly illustrate the effect of the zonal DES approach, which is shown as solid lines (__ ). The plain URANS approach, shown as dashed lines (_ _ _), exhibits a much smaller peak swirl-velocity appearing at a larger core radius $r_{c}$, giving the vortex a smeared-out appearance. This weakening of the vortices is due to the worse flow scale resolution within the URANS approach. This effect increases with wake age, as also seen by other authors (Ref. 10). The validity of the DES approach is underlined by the current experimental results shown in Fig. 11. The unfilled circles (০০০) correspond to the measured values at the same pitch angle $\Theta_{r}$, yielding slightly larger swirl velocity levels due to the higher experimental thrust

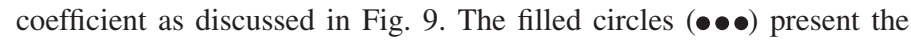
swirl velocity distribution of an experimental run with reduced angle of incidence $\left(\Theta_{r}=22.5^{\circ}\right)$. This trimmed case $\left(c_{T}=1.42 \times 10^{-2}\right)$ matches the thrust of the numerical simulations $\left(c_{T}=1.43 \times 10^{-2}\right)$ with $\Theta_{r}=24^{\circ}$ by less than $1 \%$ error. The distributions at both presented wake ages show a good agreement to the DES results. Same thrust levels result in a similar swirl velocity distribution. The agreement of the circulation and swirl velocities outside $\bar{r}_{v}=0.3$ is promising with differences less than $0.5 \%$. It is noted that the experimental results do not cover data close to the vortex center $\left(\bar{r}_{v} \rightarrow 0\right)$. This is due to voids in the PIV tracer particle distributions resulting from strong centrifugal 


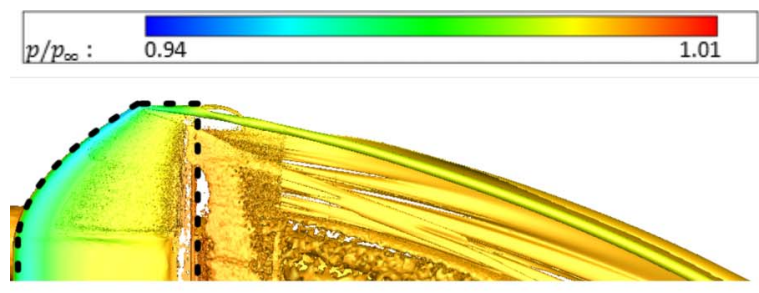

(a) $\Theta r=18^{\circ}$

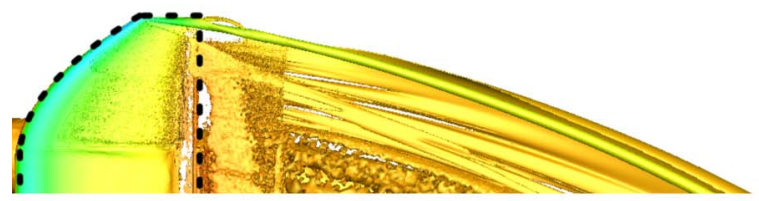

(b) $\Theta r=21^{\circ}$

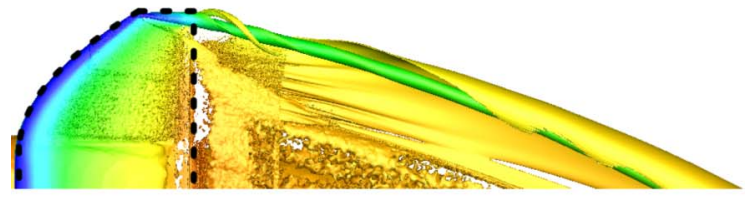

(c) $\Theta r=24^{\circ}$

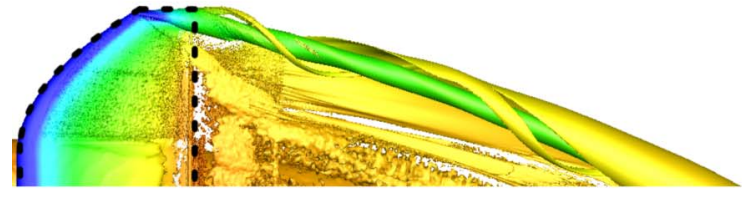

(d) $\Theta r=27^{\circ}$

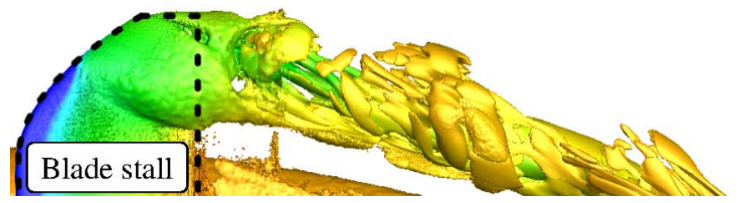

(e) $\Theta r=30^{\circ}$

Fig. 10. Closeup view on the blade-tip vortex for five different static cases.

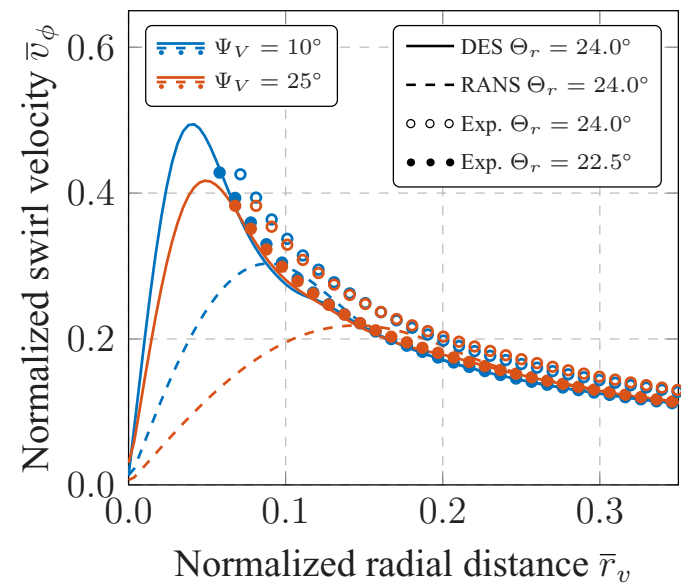

Fig. 11. Normalized swirl velocity of blade-tip vortex over radial distance with different settings (- = DES; - - - = RANS.; ००० $=$ experimental; $\bullet \bullet \bullet=$ trimmed experimental $)$.

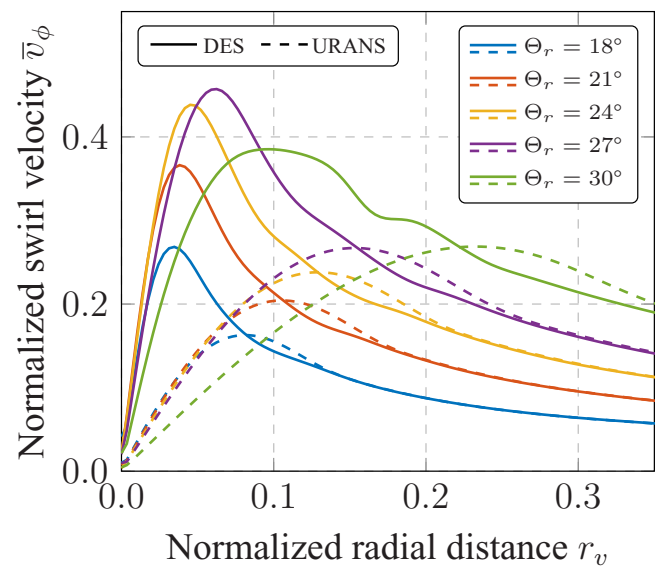

Fig. 12. Normalized swirl velocity of blade-tip vortex over radial distance for different constant pitch angles at $\Psi_{V}=20^{\circ}(-=\mathrm{DES}$; - - - = URANS).

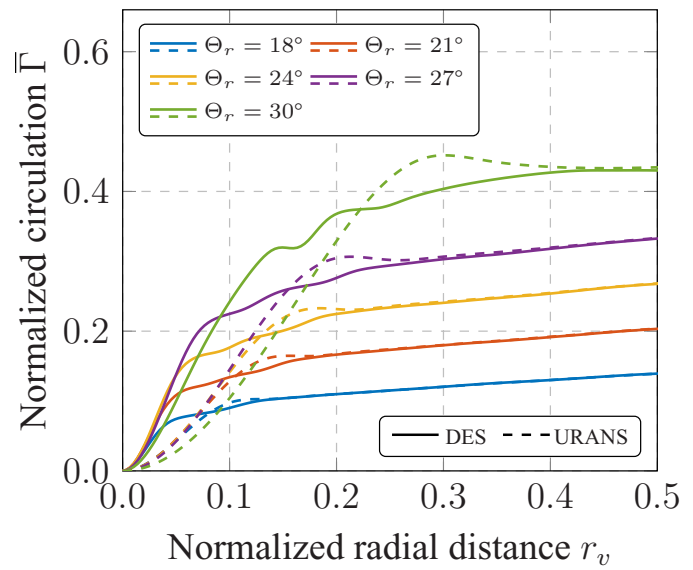

Fig. 13. Normalized circulation of blade-tip vortex over radial distance for different constant pitch angles at $\Psi_{V}=20^{\circ}(-=\mathrm{DES}$; - - - = URANS).

forces, which yields unreliable or erroneous data (see Refs. 23, 24). The voids were mapped in the PIV raw images, and the current results only show valid data outside of the voids.

Figure 12 summarizes the numerical vortex structures for different collective pitch angles between $\Theta_{r}=18^{\circ}$ and $\Theta_{r}=30^{\circ}$. Again, the lesser concentration of the vortex core region of the URANS simulations is visible. Nevertheless, both URANS and DES agree on an identical or at least very similar swirl velocity toward larger radial distances, which is due to the prescribed thrust level and, therefore, prescribed vortex circulation. The vortex strength (peak swirl strength) and core radius increase with increasing collective pitch angle $\Theta_{r}$, as could be seen in Fig. 10. The result at $\Theta_{r}=30^{\circ}$ is qualitatively different due to flow separation at this collective pitch angle.

The circulation of the blade-tip vortex can be estimated by the path integral of the velocity at a given radius, which is $\Gamma=2 \pi r \bar{v}_{\phi}$ for a circular vortex. Figure 13 shows the circulation of the blade-tip vortex over the radial coordinate. The circulation approaches a saturated value after a first quick rise in the vicinity of the vortex core, and the URANS circulation is equal to the DES circulation for $r_{v} / c>0.4$. For the sake of comparability with PIV experiments, the single value at $r_{v} / c=0.5$ is taken as the circulation of the blade-tip vortex. 


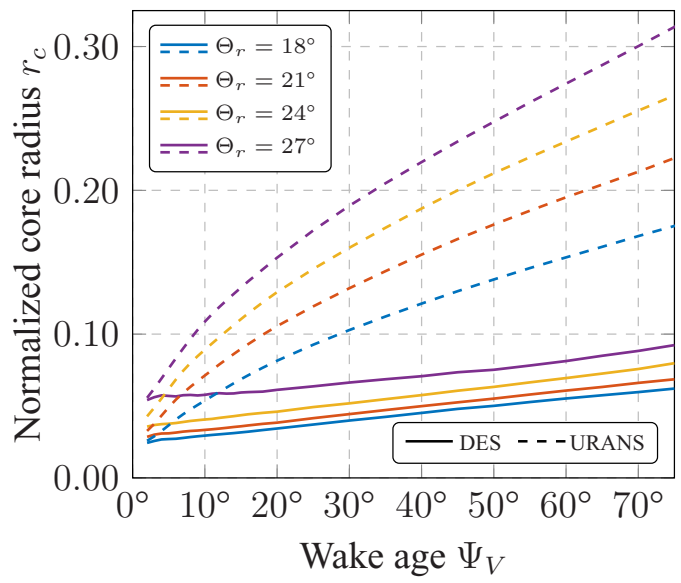

Fig. 14. Normalized radius of blade-tip vortex for different constant pitch angles over the wake age (- = DES; - - - = URANS).

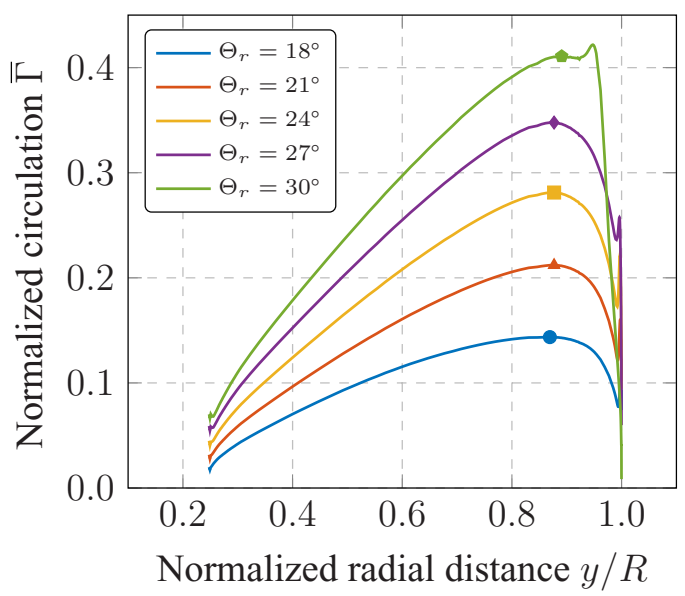

Fig. 15. Radial circulation distribution on the blade for different constant pitch angles.

The change of the vortex core radius with increasing wake age is plotted in Fig. 14. Again, the dashed curves show the significant effect of modeling and averaging all flow scales within the plain URANS approach, leading to a rapid expansion of the core size and a rapid linear increase in the core size after $\Psi_{V}=45^{\circ}$. The trend of the DES approach is a slow linear increase in core size with vortex age. The plot starts at the end of the URANS region in the DES computation, needed to ensure sufficient wall distance for the correct operation of the Smagorinsky subgrid scale model. The grid in the URANS region is constructed with very fine tetrahedral cells to keep the dissipation to a minimum.

\section{Vortex generation}

Figure 15 shows the circulation distribution on the blade, from a path integration of the surface pressure at each radial station, using the Kutta-Joukowski theorem. The circulation increases toward the tip since the increase in velocity is not fully offset by the negative linear twist, reaching a maximum around $r / R \approx 0.9$. At both the inner and outer ends, the lift and circulation are zero. All cases except for the highest angle of incidence $\Theta_{r}=30^{\circ}$ have a distinct spike close to the tip, caused by the reattachment line of the blade-tip vortex. The distinctive peak

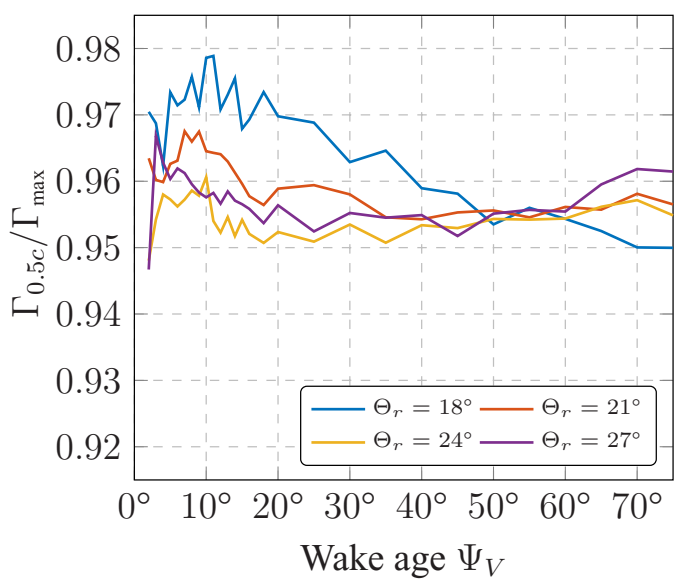

Fig. 16. Ratio of tip vortex circulation $\Gamma_{0.5 c}$ and maximum circulation on the blade $\Gamma_{\max }$ for different constant pitch angles over the wake age.

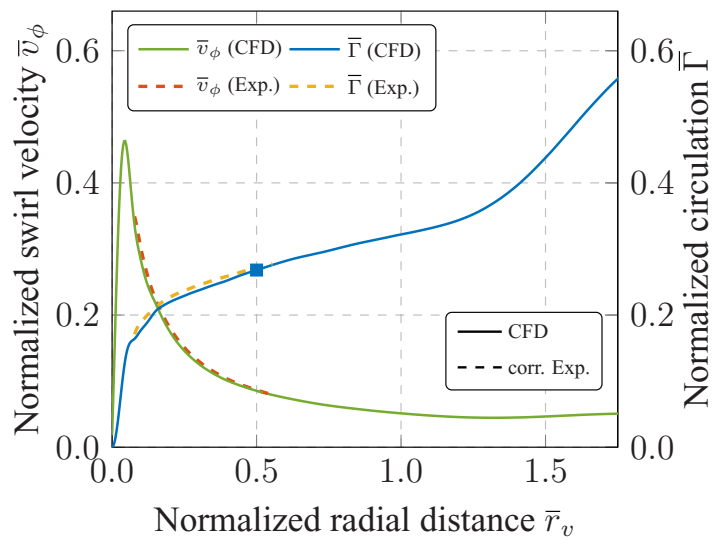

Fig. 17. Normalized swirl velocity and normalized circulation of blade-tip vortex over radial distance $\left(\Theta_{r}=24^{\circ}, \Psi_{V}=15^{\circ}\right)$.

is further inboard for $\Theta_{r}=30^{\circ}$ case, as the separation/vortex region expands to cover nearly the entire blade tip, as also seen in Fig. 10(e).

In Fig. 15, the maximum circulation is marked with a symbol. The rollup process of a blade-tip vortex depends on the sign of the gradient of the circulation, with a positive gradient inboard of the maximum and a negative gradient outboard of the maximum circulation. All the circulation outboard of the maximum feeds the blade-tip vortex, due to the negative gradient, with the remaining circulation feeding the root vortex. This analysis allows for a comparison of the blade circulation outboard of this maximum and the circulation $\Gamma_{0.5 c}$ of the blade-tip vortex at $r_{v} / c=0.5$. The ratio of both is shown in Fig. 16, showing that about $95 \%$ of the blade circulation can be identified as present in the tip vortex.

Figure 17 shows a comparison of numerical and corrected experimental blade-tip vortex circulations over the radial coordinate. The trend in the CFD data inboard of $r_{v} / c=0.5$ shows a linear increase up to $r_{v} / c \approx 1.2$. Beyond this distance, there is a rapid increase which is not related to the tip vortex, but to an artifact of the circulation computation stretching to the shear flow between the rotor wake and the external flow. The analysis of the blade circulation allows for differentiation between the near field and far field of a vortex, where $100 \%$ of the blade circulation is present in the "vortex." The complete blade circulation is found within $r_{v} / c=0.6$, and thus this area is the near field of the vortex. Figure 17 also shows a comparison of corrected experimental and 


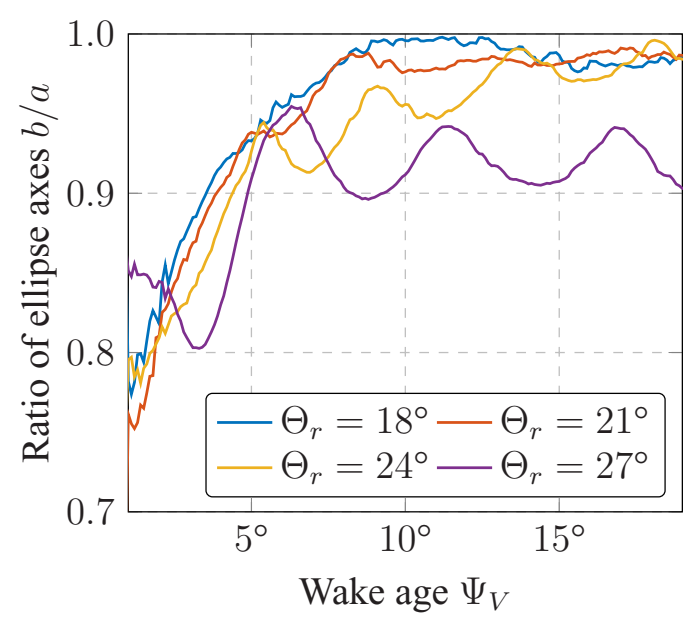

Fig. 18. Ratio of the axes of the ellipse of the vortex core for different angle of incidences.

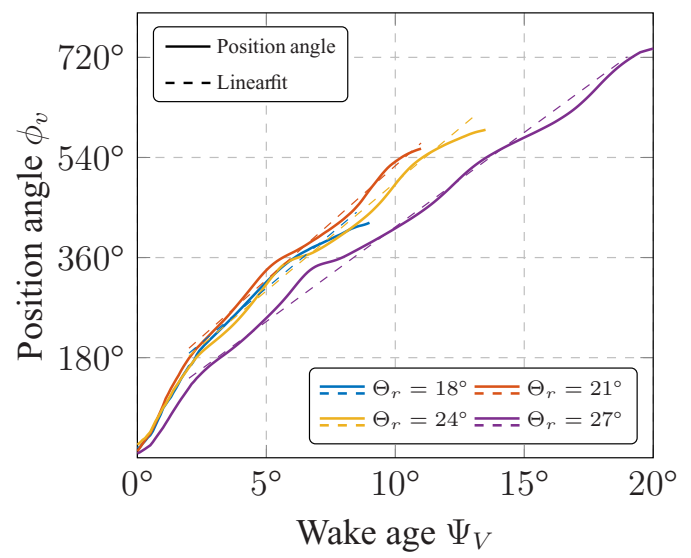

Fig. 19. Position angle of ellipse.

numerical normalized swirl velocity, revealing a good agreement between both results.

\section{Vortex propagation}

As indicated in Fig. 6, the vortex can be fitted as an ellipse with major and minor axes $a$ and $b$. After being generated on the blade, the vortex is quite elliptical, as seen in Fig. 18, and becomes rounder with time. In the case with the lowest angle of incidence (_ $)$, the rounding of the ellipse progresses most quickly, reaching a ratio of $b / a=0.98$ before $\Psi_{V}=10^{\circ}$, which is 1.5 chord lengths behind the rotor blade, consistent with Refs. 2-4. Interestingly, there is a pulsation noticeable in the case with $\Theta_{r}=24^{\circ}$ and $\Theta_{r}=27^{\circ}$, repeating every $\Delta \Psi_{V}=4^{\circ}$ and $5^{\circ}$, which is of the same order as the solid-body rotation speed. Since the radius is fairly constant in this area and a pure rotation results in a constant ratio, the change of the ratio $b / a$ occurs due to an overlaying secondary motion. A complete revolution of the shape is reached after two pulses, as $b / a$ is symmetric after half a revolution. For $\Theta_{r}=30^{\circ}$, the vortex shape is more complex and does not lend itself to an elliptical vortex fit.

Figure 19 gives insight into the rotation of the vortex core by displaying the position angle $\phi_{v}$ of the fitted ellipse. An increase in $\phi_{v}$ implies a counterclockwise rotation, which is the characteristic rotational direction of the vortex core. With increasing wake age, the vortex-core shape

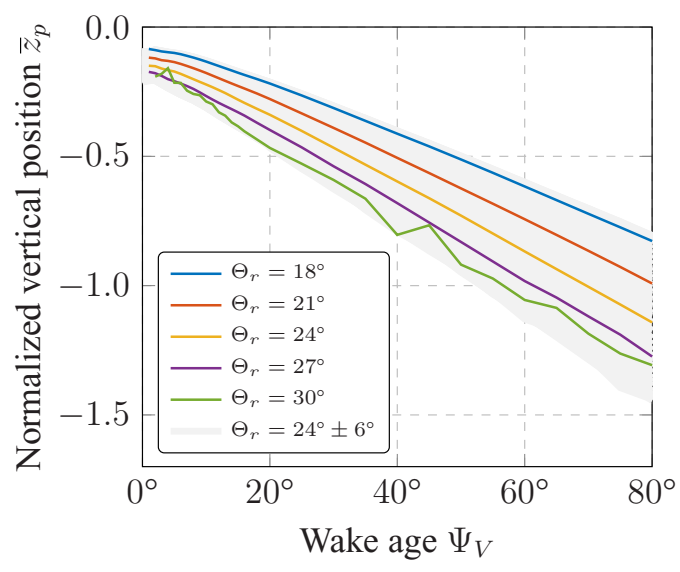

Fig. 20. Vertical position of the blade-tip vortex for different angle of incidences over the wake age (steady and unsteady cases).

approaches a circle, and the angle detection is terminated when an angle can no longer be calculated. Linear fits result in slopes $\delta \phi_{v} / \delta \Psi_{V}$, indicating a frequency of the shape rotation $f \approx 944 \mathrm{~Hz}$, which is 40 times higher than the rotational frequency of the rotor. The rotational frequency $f=v_{\phi, \max } /\left(2 \pi r_{c}\right)$ of the vortex core can be computed with the peak swirl-velocity and core radius. This core rotation frequency describes the frequency or reciprocal time of a particle performing a circle on the core radius with a circumference of $2 \pi r_{c}$ with the prescribed peak swirl-velocity $v_{\phi, \max }$. It is often represented as a solid-body rotation and is on the order of $1500-2000 \mathrm{~Hz}$, roughly double the rotation frequency computed from following the elliptical shape of the vortex.

\section{Vortex-core position}

Both following plots separately show the vertical and horizontal displacement over the wake age for different angles of incidences. The vertical vortex-core position is displayed in Fig. 20, showing that the higher collective angles lead to higher lift and faster downward convection of the vortex. Interestingly, the unsteady pitching case for $\Theta_{r}=24^{\circ}-6^{\circ} \cos (2 \pi t / T)$ covers the range indicated by the cases with collective pitch only, filling out the region under $\Theta_{r}=30^{\circ}$, since for the dynamic pitching, no stall is seen at this maximum angle of attack. For the pitching rotor, the thrust coefficient is roughly the same as that for $\Theta_{r}=24^{\circ}$, so it can be seen that the vertical position of the blade-tip vortex is a function of the instantaneous blade lift, rather than the rotor thrust. The $z$-position of the blade-tip vortex for $\Theta_{r}=21^{\circ}$ is one chord length $\left(\Delta z_{p} / c \approx 1\right)$ below the rotor disk at $\Psi_{V}=80^{\circ}$. The vertical vortex propagation rate was between three to five chord lengths per revolution.

The opposite is seen for the horizontal blade-tip vortex position, as seen in Fig. 21. In the cases without a cyclic pitch, the vortex moves more strongly toward the blade root with increasing thrust, corresponding to the stronger contraction of the stream tube of the rotor wake required by conservation of mass. The blade-tip vortex is at $\Delta y_{p} / c \approx 0.8$ inboard for $\Theta_{r}=21^{\circ}$ and $\Psi_{V}=80^{\circ}$, and in the first quadrant the inboard horizontal propagation rates were between two and four chord lengths per revolution. In contrast, the stream tube contraction in the case with cyclic pitch is, on average, the same as for $\Theta_{r}=24^{\circ}$, and clearly, the changes in local thrust do not affect the contraction as much as for constant pitch. The location of the vortices in the dynamic pitching case, thus, occupies a smaller band around $\Theta_{r}=24^{\circ}$. 


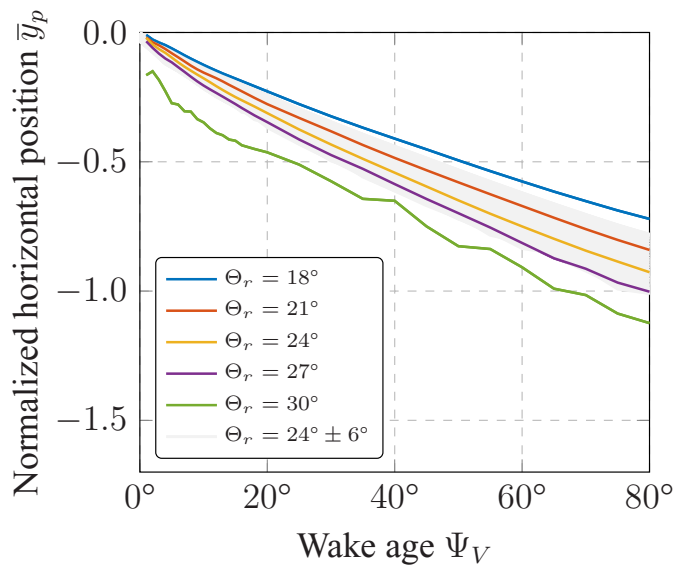

Fig. 21. Horizontal position of the blade-tip vortex for different angle of incidences over the wake age (Steady and unsteady cases).

\section{Unsteady vortex}

The dynamically pitching rotor produces flow, which is qualitatively similar to that seen during the test cases with a constant pitch. Figure 22 shows the $\lambda_{2}$ isosurfaces of the flow around the blade tip, analogous to the static results shown in Fig. 10. The sole exception to the qualitative similarity is that while there is separated flow on the blade tip for static $\Theta_{r}=30^{\circ}$, the flow remains fully attached for the dynamically pitching case the flow remains fully attached.

Figure 23 shows the static vertical position of the blade-tip vortex and the vertical positions of their corresponding counterparts during the unsteady motion. Here the unsteady lines are not connected with the numerical or isochronic approach ( line in Fig. 5) but with their

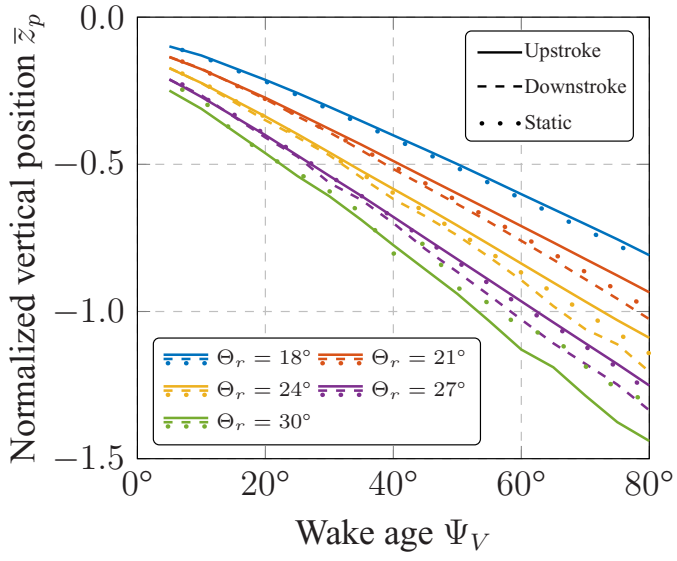

Fig. 23. Vertical position of blade-tip vortex against wake age (curves display constant position approach,

geodesic position (-0 line in Fig. 5). This means that the vortex position is followed in time after it is produced by the blade tip and convects away. By assuming that the vortex slice stayed at a constant azimuthal angle in the laboratory frame, the propagation can easily be followed. At the intermediate angles $\left(\Theta_{r}=\left[21^{\circ}, 24^{\circ}, 27^{\circ}\right]\right)$, the static vortices are surrounded by the unsteady vortices, with a nearly equal offset to each side, and vortices which were created during the upstroke motion have less downward convection than those created during the downstroke motion. At the minimum angle $\left(\Theta_{r}=18^{\circ}\right)$ the static vortex is roughly equivalent to the dynamic vortex, and at the maximum angle $\left(\Theta_{r}=30^{\circ}\right)$ the dynamic vortex is propagated further downwards due to the higher lift in the unstalled flow. At the extrema, the upstroke/downstroke lines fall on top of each other.

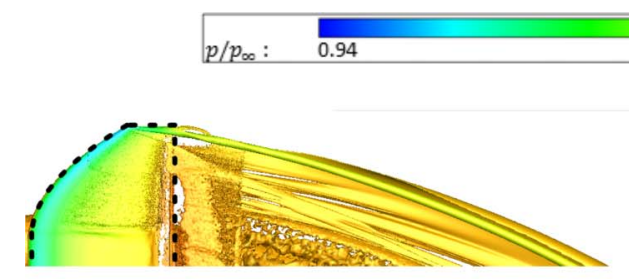

(a) $\Theta r=18^{\circ}$

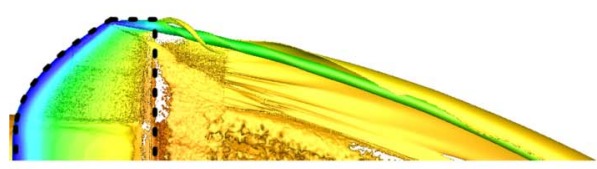

(c) $\Theta r=24^{\circ} \uparrow$

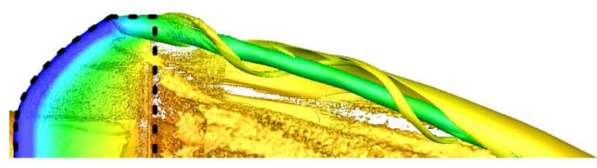

(e) $\Theta r=30^{\circ}$

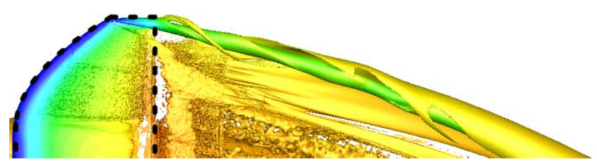

(g) $\Theta r=24^{\circ} \downarrow$

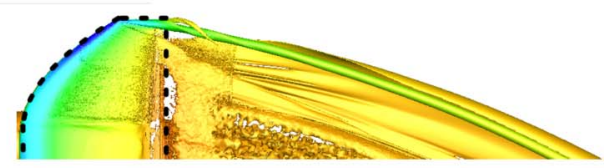

(b) $\Theta r=21^{\circ} \uparrow$

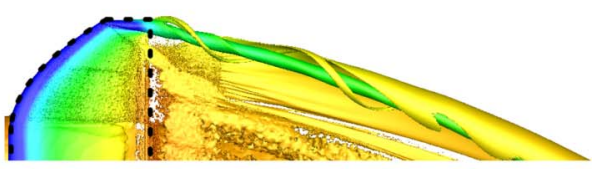

(d) $\Theta r=27^{\circ} \uparrow$

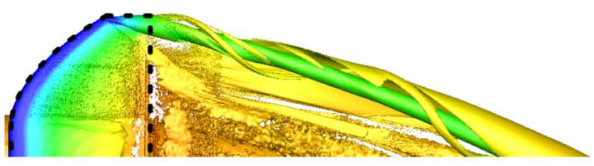

(f) $\Theta r=27^{\circ} \downarrow$

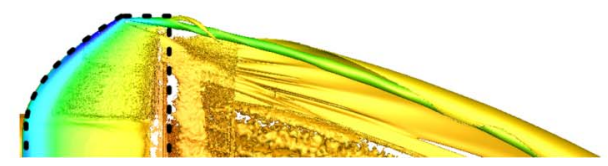

(h) $\Theta r=21^{\circ} \downarrow$

Fig. 22. Closeup view of the blade-tip vortex for eight different cases during an unsteady pitch motion. 


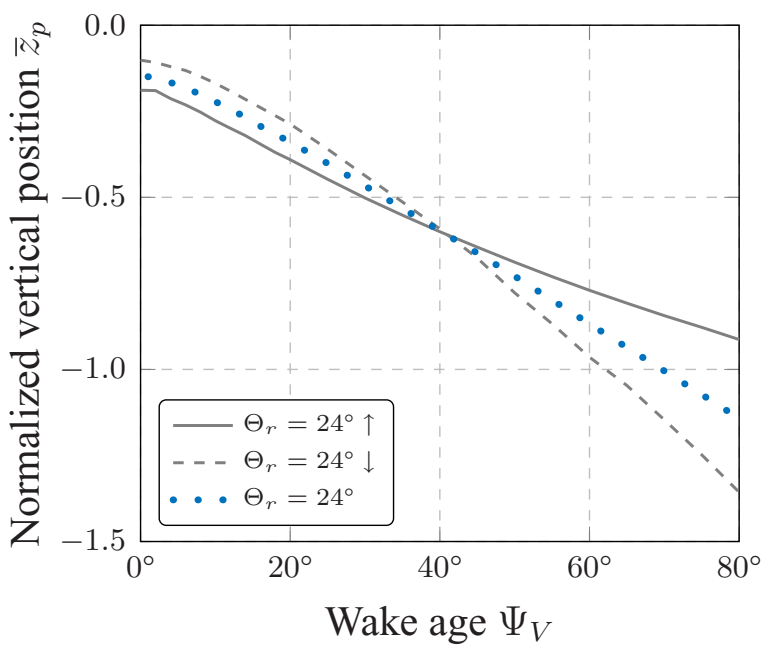

(a) Vertical position

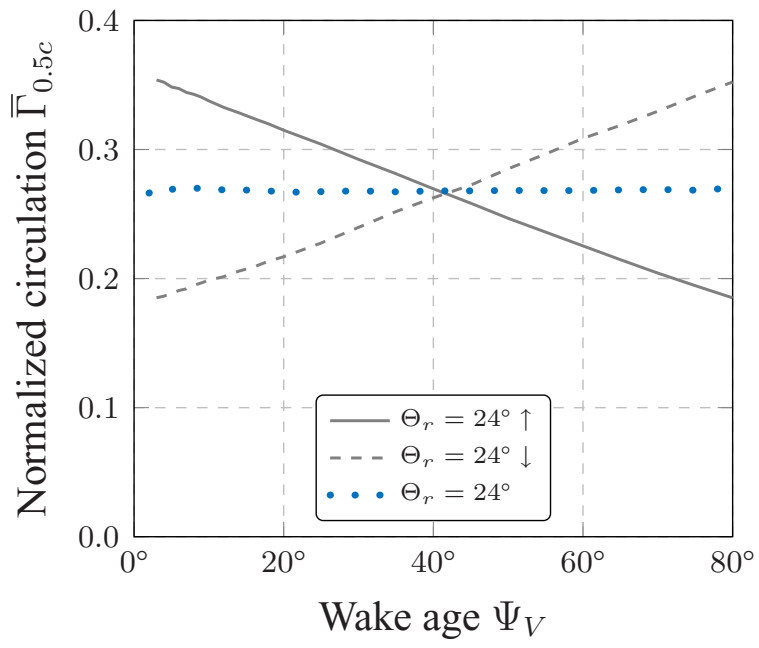

(c) Circulation

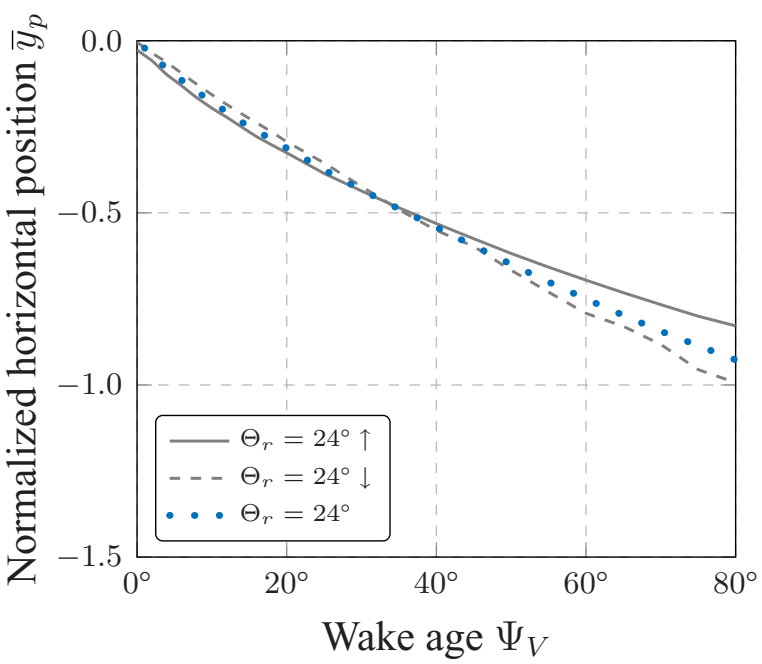

(b) Horizontal position

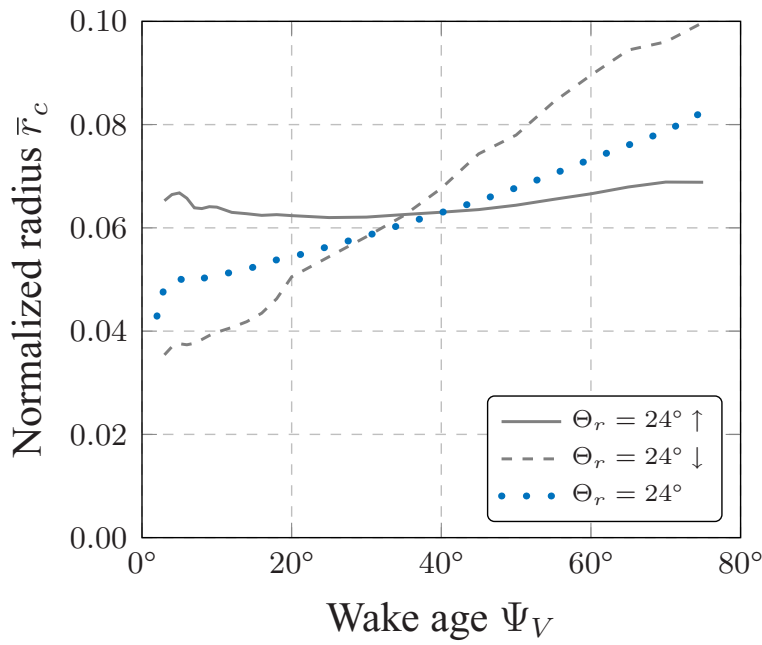

(d) Radius

Fig. 24. Vertical (a), horizontal (b) position, normalized circulation (c), and radius (d) of unsteady blade-tip vortex against wake age (unsteady curves display constant time approach, $\rightleftharpoons$ line in Fig. 5).

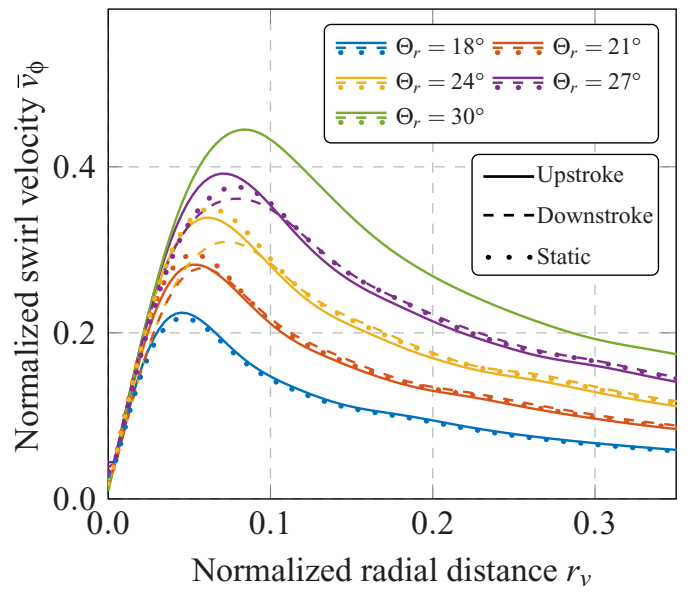

Fig. 25. Normalized swirl velocity of blade-tip vortex over radial distance for different angles of incidence at $\Psi_{V}=40^{\circ}(-=$ upstroke; - - = downstroke; $\cdots=$ static).

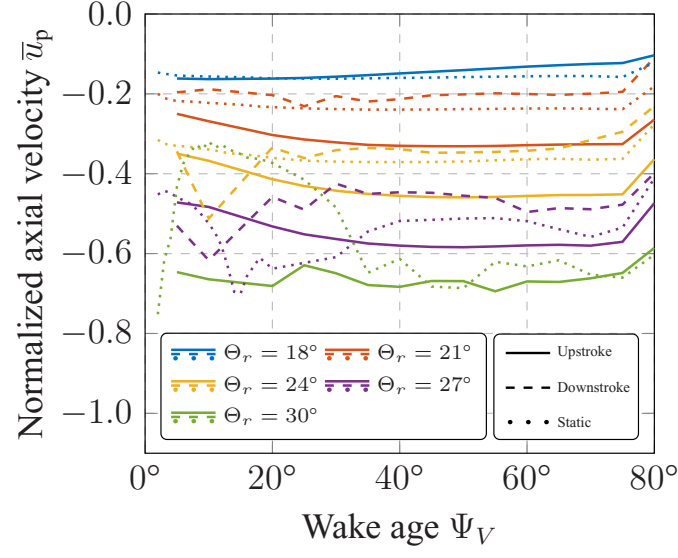

Fig. 26. Normalized axial velocity inside the blade-tip vortex for different instants of time in the pitching cycle over the wake age (curves display constant position approach, - = upstroke; - - - = downstroke). 


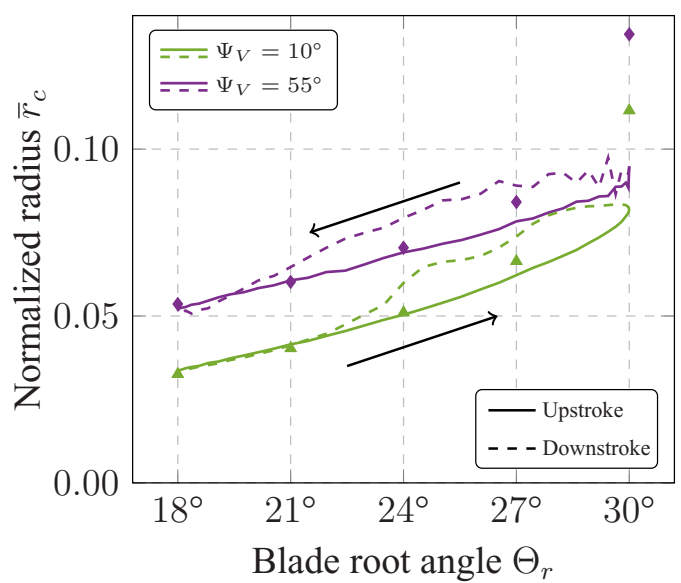

Fig. 27. Normalized radius of blade-tip vortex throughout an entire pitching cycle for two wake ages (curves display constant wake age, $\bullet \bullet$ line in Fig. 5, - = upstroke; - - = downstroke); $\bullet=$ static).

The result above suggests strongly that the unsteady vortex can be modeled as an assembly of the static vortex states. In Fig. 24, the different vortex parameters are extracted in the case where the vortex at $\Psi_{V}=45^{\circ}$ in each snapshot was produced when the rotor blade had the instantaneous pitch angle of $\Theta_{r}=24^{\circ}$, and has thereafter been convected to that position, assuming that each slice remains at a constant azimuthal angle in the laboratory frame. The difference in the vertical position (a) and horizontal (b) position are not significantly high. The circulation (c) is constant for the static case and a steep decrease during the upstroke motion. A positive gradient results due to the downstroke motion. The increase of the radius (d) is diminished during the upstroke motion and enlarged during the downstroke motion. It can be seen that within the small errors produced by the lift hysteresis, that the position, circulation, and radius of the vortex are very similar for upstroke, downstroke, and static collective pitch at $\Psi_{V}=45^{\circ}$. For the dynamic cases, the vortex changes, moving away from the crossing point associated with the nominal $\Theta_{r}=24^{\circ}$ point at $\Psi_{V}=45^{\circ}$, depending on whether the vortex strength is increasing or decreasing with time. This suggests that an approach that tracks the blade lift distribution at the moment of production and the aging of each slice through the propagating vortex should have a good chance of producing a physically correct vortex.

Figure 25 shows the normalized swirl velocity for dynamic and static test cases at a wake age of $\Psi_{V}=40^{\circ}$. The dynamic results have been matched with the static test cases according to the angle of incidence during vortex creation. It can be seen that, disregarding the small hysteresis in the unsteady case, the dynamic vortex shapes are well predicted by the static vortex shapes associated with the same angle of attack. Similarly, Fig. 26 shows the normalized axial velocity $u_{\mathrm{p}} / U_{\text {tip }}$ occurring in the center of the blade-tip vortex. Negative values characterize a reduction in flow speed relative to the blade as in a wake velocity deficit region. The axial velocity magnitude increases (in the negative direction) with an increasing angle of incidence, which is equivalent to a higher wake deficit. The downstroke situations have a weaker wake deficit. In contrast with the earlier investigation by Goerttler et al. (Ref. 25) using URANS with a two-bladed rotor, which noted backward jetting associated with vortex breakdown occurring during high thrust and blade stall, here all axial velocities are negative. This is due to the lack of blade dynamic stall in the test cases investigated in this paper.

Figure 27 displays the radius of the vortex core throughout an entire pitching cycle for two wake ages. The radius grows with increasing angle of incidence and is bigger, the higher the wake age. The static results are close to the values obtained during the upstroke (__ motion. After the maximum angle of incidence, the radius decreases again but remains at a higher level than on the upstroke, which agrees with the observation by Mohamed et al. (Ref. 10) for a pitching blade.

\section{Variation of the Reynolds number}

Scaling rotor vortices is a particular problem and relevant since many investigations, for example Ref. 37, are only practicable in model scale, and the results must be scaled. As a simple test, all parameters of the simulation were held constant, except the freestream pressure/density, which were increased by an order of magnitude. This approach increases the Reynolds number of the flow field by an order of magnitude as well.

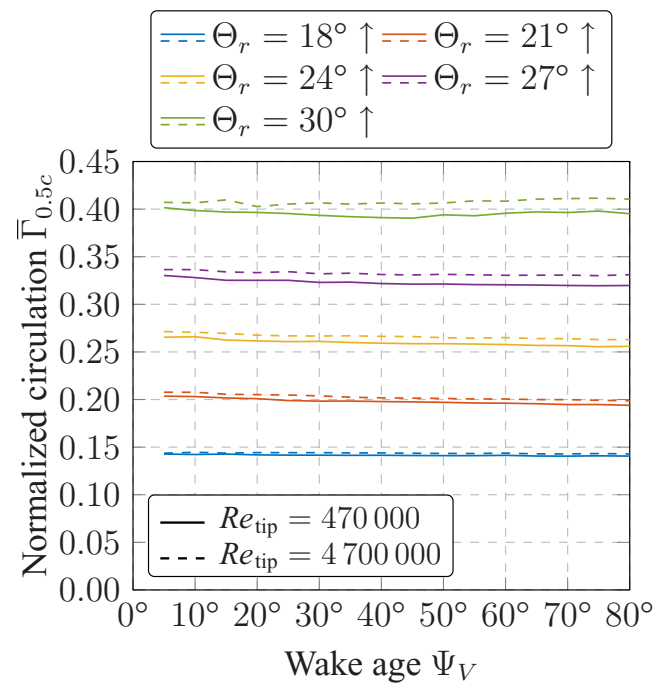

(a) Circulation

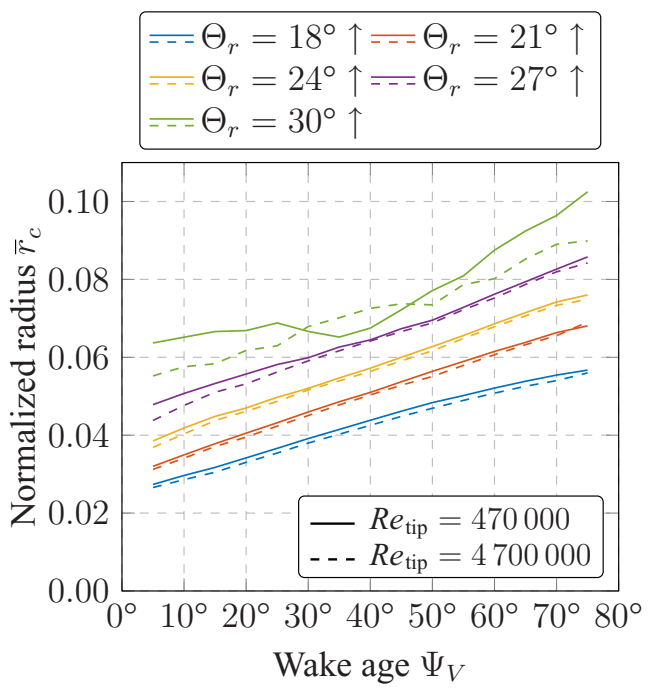

(b) Radius

Fig. 28. Normalized circulation and radius of blade-tip vortex for different instants of time in the pitching cycle over the wake age (curves display constant position approach, $0 \circ$ line in Fig. $5,-=R e_{\text {tip }}=470,000 ;--=R e_{\text {tip }}=4,700,000$ ). 
Figure 28 shows the results, which are each normalized with the relevant freestream density. Figure 28(a) displays a slightly higher circulation (2-4\%) of the blade-tip vortex for the higher Reynolds number, which appears to be primarily due to the slightly higher thrust coefficient for the same pitching motion due to the change in boundary layer shape. Despite this, the vortex core radius is smaller for the higher Reynolds number, reducing in size by $2-5 \%$. These results indicate that the Reynolds number is not a useful scaling parameter for vortices. Other approaches, which include the thrust coefficient, seem more promising, like the vortex Reynolds number.

\section{Conclusions}

A numerical investigation of the four-bladed RTG rotor in axial flow with cyclic and collective pitch test cases was performed. The use of a fine grid with a DES solver in the vortex region resulted in computed vortices, which are comparable with those measured experimentally, and which do not display the rapid diffusion of vorticity seen for vortices computed with RANS solvers. The numerical simulations were validated with experimental collective cases allowing for a novel further exploration of an unsteady case. The main conclusions are as follows:

1) Similar thrust levels lead to a very good agreement of the swirl velocity distribution between numerical and experimental data.

2) The unsteady vortex can be modeled as an assembly of the steady vortex states. The instantaneous vortex section produced by the rotor blade is comparable in the static and dynamic cases and can be followed as it convects downstream.

3 ) Increasing the Reynolds number by a factor of 10 did not result in a significant difference in the vortex parameters circulation and radius. The Reynolds number of the flow field is not a useful scaling parameter for vortices.

4) The vorticity in the vortex is well predicted by the maximum circulation on the blade. The blade-tip vorticity is contained within $r_{v} / c=0.6$, and this region can be defined as the near field of the vortex.

5 ) The error from misidentifying the vortex center position on the shape, radius, and swirl velocity is small. A discrepancy in position of up to $10 \%$ reduces the radius by less than $0.5 \%$ and swirl velocity by less than $1 \%$.

6) The elliptical vortex shape rotates with approximately half the solid-body rotation speed of the core. Two orthogonal cuts are sufficient to determine the average radius by an error less than $3 \%$.

7) The rotation of the vortex-core shape is influenced by the secondary vortex and therefore rotates with a slower frequency than the possible solid body rotation.

\section{Acknowledgments}

Computing time was provided by the Gauss Centre for Supercomputing e.V. (GCS, www.gauss-centre.eu) on the GCS supercomputer SuperMUC at the Leibniz Supercomputing Centre (LRZ, www.lrz.de) under project pr53fi. This work was part of the DLR project FAST-Rescue. Dr. Kurt Kaufmann is thanked for his invaluable assistance.

\section{References}

\footnotetext{
${ }^{1}$ Jeong, J., and Hussain, F., "On the Identification of a Vortex," Journal of Fluid Mechanics, Vol. 285, 1995, pp. 69-94, DOI: 10.1017/S0022112095000462.

${ }^{2}$ Chow, J., Zilliac, G., and Bradshaw, P., "Mean and Turbulence Measurements in the Near Field of a Wingtip Vortex," AIAA Journal, Vol. 35 , (10), 1997, pp. 1561-1567, DOI: 10.2514/2.1.
}

${ }^{3}$ Birch, D., Lee, T., Mokhtarian, F., and Kafyeke, F., "Structure and Induced Drag of a Tip Vortex," Journal of Aircraft, Vol. 41, (5), 2004, pp. 1138-1145, DOI: 10.2514/1.2707.

${ }^{4}$ Devenport, W., Rife, M., Liapis, S., and Follin, G., "The Structure and Development of a Wing-Tip Vortex," Journal of Fluid Mechanics, Vol. 312, 1996, pp. 67-106, DOI: 10.1017/S0022112096001929.

${ }^{5}$ Dacles-Mariani, J., Zilliac, G., Chow, J., and Bradshaw, P., "Numerical/Experimental Study of a Wingtip Vortex in the Near Field," AIAA Journal, Vol. 33, (9), 1995, pp. 1561-1568, DOI: 10.2514/3.12826.

${ }^{6}$ Lombard, J., Moxey, D., and Sherwin, S., "Implicit Large-Eddy Simulation of a Wingtip Vortex," AIAA Journal, Vol. 54, (2), 2016, pp. 506-518, DOI: 10.2514/1.J054181.

${ }^{7}$ Gray, R., "An Aerodynamic Analysis of a Single-Bladed Rotor in Hovering and Forward Flight as determined by Smoke Studies on the Vorticity Distribution in the Wake," Princeton University, TR-356, Princeton, NJ, 1957.

${ }^{8}$ Leishman, J., Baker, A., and Coyne, A., "Measurements of Rotor Tip Vortices Using Three-Component Laser Doppler Velocimetry," Journal of the American Helicopter Society, Vol. 41, (4), October 1996, pp. 342 353, DOI: 10.4050/JAHS.41.342.

${ }^{9}$ Heineck, J., Yamauchi, G., Wadcock, A., Lourenco, L., and Abrego, A., "Application of Three-Component PIV to a Hovering Rotor Wake," Proceedings of the American Helicopter Society 56th Annual Forum, Virginia Beach, VA, May 2-4, 2000.

${ }^{10}$ Mohamed, K., Nadarajah, S., and Paraschivoiu, M., "DetachedEddy Simulation of a Wing Tip Vortex at Dynamic Stall Conditions," Journal of Aircraft, Vol. 46, (4), 2009, pp. 1302-1313, DOI: 10.2514/1.40685.

${ }^{11}$ Garmann, D., and Visbal, M., "Unsteady Evolution of the Tip Vortex on a Stationary and Oscillating NACA0012 Wing," Proceedings of the 54th AIAA Aerospace Sciences Meeting, San Diego, CA, January 4-8, 2016, DOI: $10.2514 / 6.2016-0328$.

${ }^{12}$ Garmann, D., and Visbal, M., "Further Investigations of the Tip Vortex on an Oscillating NACA0012 Wing," Proceedings of the 46th AIAA Fluid Dynamics Conference, Washington, D.C., June 13-18, 2016, DOI: 10.2514/6.2016-4343.

${ }^{13}$ Garmann, D., and Visbal, M., "Investigation of the Unsteady Tip Vortex Structure on a NACA0012 Wing at Fixed Incidence," Proceedings of the 55th AIAA Aerospace Sciences Meeting, Grapevine, TX, January 9-13, 2017, DOI: 10.2514/6.2017-1002.

${ }^{14}$ Duraisamy, K., Ramasamy, M., Baeder, J., and Leishman, J., "HighResolution Computational and Experimental Study of Rotary-Wing Tip Vortex formation," AIAA Journal, Vol. 45, (11), 2007, pp. 2593-2602, DOI: $10.2514 / 1.26575$.

${ }^{15}$ Lietzau, Z., Ramasamy, M., Jain, R., Leishman, J., and Ekaterinas, J., "Comparison of Advanced RANS Modeling with Dual-Plane PIV Measurements for a Hovering Rotor," Journal of the American Helicopter Society, 63, 022003 (2018), DOI: 10.4050/JAHS.63.022003.

${ }^{16}$ Platzer, S., Rauleder, J., and Hajek, M., "Experimental and Computational Investigation on Rotor Blades with Spanwise Blowing," Proceedings of the 42nd European Rotorcraft Forum, Lille, September 6-8, 2016.

${ }^{17}$ Jain, R., "Sensitivity Study of High-Fidelity Hover Predictions on the Sikorsky S-76 Rotor," Journal of Aircraft, Vol. 55, (1), 2017, pp. 78-88, DOI: 10.2514/1.C034076.

${ }^{18}$ Richez, F., "Analysis of Dynamic Stall Mechanisms in Helicopter Rotor Environment," Journal of the American Helicopter Society, 63, 022006 (2018), DOI: 10.4050/JAHS.63.022006.

${ }^{19}$ Chaderjian, N., "Navier-Stokes Simulation of UH-60A Rotor/ Wake Interaction Using Adaptive Mesh Refinement," Proceedings of the AHS 73rd Annual Forum, Fort Worth, TX, May 9-11, 2017. 
${ }^{20}$ Jayaraman, B., and Potsdam, M., "Effect of Fuselage and Wind Tunnel Wall on Full-Scale UH-60A Rotor Tip Vortex Prediction," Proceedings of the 34th AIAA Applied Aerodynamics Conference, Washington, DC, June 13-17, 2016, DOI: 10.2514/6.2016-3131.

${ }^{21}$ Wolf, C., Merz, C., Richter, K., and Raffel, M., "Tip-Vortex Dynamics of a Pitching Rotor Blade-Tip Model," AIAA Journal, Vol. 54, (10), 2016, pp. 2947-2960, DOI: 10.2514/1.J054656.

${ }^{22}$ Kaufmann, K., Wolf, C., Merz, C., and Gardner, A., "Numerical Investigation of Blade-Tip-Vortex Dynamics," Proceedings of the 17th ONERA-DLR Aerospace Symposium, Aussois, France, June 7-9, 2017.

${ }^{23}$ Wolf, C., Braukmann, J., Stauber, W., Schwermer, T., and Raffel, M., "The Tip Vortex System of a Four-Bladed Rotor in Dynamic Stall Conditions," Journal of the American Helicopter Society, 64, 022005 (2019), DOI: 10.4050/JAHS.64.022005.

${ }^{24}$ Braukmann, J., Goerttler, A., Wolf, C., and Raffel, M., "Blade-Tip Vortex Characterization of a Rotor under Static and Cyclic Pitch Conditions Using BOS and PIV," Proceedings of the 57th AIAA Aerospace Sciences Meeting, San Diego, CA, January 7-11, 2019.

${ }^{25}$ Goerttler, A., Braukmann, J., Schwermer, T., Gardner, A., and Raffel, M., "Tip-Vortex Investigation on a Rotating and Pitching Rotor Blade," Journal of Aircraft, Vol. 55, (5), 2018, pp. 1792-1804, DOI: 10.2514/1.C034693.

${ }^{26}$ Menter, F., "Zonal Two Equation k- $\omega$ Turbulence Models for Aerodynamic Flows," AIAA 93-2906, Proceedings of the 23rd AIAA Fluid Dynamics, Plasma Dynamics, and Lasers Conference, Orlando, FL, July 6-9, 1993.

${ }^{27}$ Travin, A., Shur, M., Strelets, M., and Spalart, P., "Advances in LES of Complex Flows," Fluid Mechanics and Its Applications, Kluwer Academic Publishers, Boston, MA, 2002, DOI: 10.1007/0-306-48383$1 \_16$.

${ }^{28}$ Smagorinsky, J., "General Circulation Experiments with the Primitive Equations: 1. The Basic Experiment," Mon. Weather Review, Vol. 91, (3), 1963, pp. 99-164.
${ }^{29}$ Chaderjian, N., and Buning, P., "High Resolution Navier-Stokes Simulation of Rotor Wakes," Proceedings of the AHS 67th Annual Forum, Virginia Beach, VA, May 3-5, 2011.

${ }^{30}$ Schwermer, T., Gardner, A., and Raffel, M., "A Novel Experiment to Understand the Dynamic Stall Phenomenon in Rotor Axial Flight," Journal of the American Helicopter Society, 64, 012004 (2019), DOI: 10.4050/JAHS.64.012004.

${ }^{31}$ Letzgus, J., Gardner, A., Schwermer, T., Keßler, M., and Krämer, E., "Investigation of Dynamic Stall on a Rotor with Cyclic Pitch Control," Proceedings of the 43rd European Rotorcraft Forum, Milan, Italy, September 12-15, 2017.

${ }^{32}$ Betz, A., "Verhalten von Wirbelsystemen," Zeitschrift fuer Angewandte Mathematik und Mechanik, Vol. 12, (3), 1932, pp. 164 174.

${ }^{33}$ Rossow, V., "On the Inviscid Rolled-Up Structure of Lift-Generated Vortices," Journal of Aircraft, Vol. 10, (11), 1973, pp. 647-650, DOI: 10.2514/3.60277.

${ }^{34}$ Bauknecht, A., Ewers, B., Schneider, O., and Raffel, M., “Aerodynamic Results from the STAR Hover Test: An Examination of the Active Twist Actuation," Proceedings of the 41st European Rotorcraft Forum, Munich, September 1-4, 2015.

${ }^{35}$ Ramasamy, M., Johnson, B., Huismann, T., and Leishman, J., "Digital Particle Image Velocimetry Measurements of Tip Vortex Characteristics Using an Improved Aperiodicity Correction," Journal of the American Helicopter Society, 54, 012004 (2009), DOI: 10.4050/JAHS.54.012004.

${ }^{36}$ Kaufmann, K., Wolf, C., Merz, C., and Gardner, A., "Numerical Investigation of Blade-Tip-Vortex Dynamics," CEAS Aeronautical Journal, Vol. 9, (1), 2018, pp. 373-386, DOI: 10.1007/s13272-018-0287-2.

${ }^{37}$ Schwarz, C., Bauknecht, A., Mailänder, S., and Raffel, M., "Wake Characterization of a Free-Flying Model Helicopter in Ground Effect," Journal of the American Helicopter Society, 64, 012010 (2019), DOI: 10.4050/JAHS.64.012010. 